\title{
EL MODELO DE CALIDAD DE VIDA Y APOYOS: LA UNIÓN TRAS VEINTICINCO AÑOS DE CAMINOS PARALELOS ${ }^{1}$
}

\section{The Quality of Life Supports Model: Twenty-Five Years of Parallel Paths Have Come Together}

Miguel Ángel Verdugo Alonso

INICO-Universidad de Salamanca

verdugo@usal.es

Robert L. SCHALOCK

University of Nebraska

Laura E. Gómez SÁncheZ

Universidad de Oviedo

Recepción: 20 de enero de 2021

Aceptación: 22 de enero de 2021

RESUMEN: El artículo presenta la integración operativa de los paradigmas de calidad de vida y apoyos en un modelo conjunto denominado Modelo de Calidad de Vida y Apoyos (MOCA) que integra características significativas de la transformación actual en el campo de las discapacidades intelectuales y del desarrollo. Estas características abarcan un enfoque holístico e integrado, centrado en los derechos humanos y legales, que sirve de base para tomar las decisiones sobre servicios y apoyos en las limitaciones significativas de las principales áreas de actividad de la vida, con un énfasis en los apoyos individualizados proporcionados dentro de ambientes inclusivos de la comunidad y que promueva la evaluación de resultados. Los contenidos de este artículo incluyen: (a) los cuatro elementos del MOCA: valores fundamentales, dimensiones de calidad de vida individual y familiar, sistemas de apoyo y condiciones facilitadoras; (b) cómo se puede utilizar el MOCA como marco para la provisión de apoyos, la evaluación de resultados centrada en la persona, la transformación de la organización y el cambio de sistemas; y

1 Financiación: este trabajo ha sido financiado por el Ministerio de Ciencia e Innovación (Agencia Estatal de Investigación; AEI) de España (PID2019-110127GB-I00/AEI/10.13039/501100011033; PID2019105737RB-I00/AEI/10.13039/501100011033). 
(c) cómo el MOCA es esencial para el cambio de paradigma actual en el campo de las discapacidades intelectuales y del desarrollo.

Palabras Clave: discapacidad intelectual; discapacidades del desarrollo; calidad de vida; apoyos; modelo; paradigma; modelo de calidad de vida y apoyos.

AвSTRACT: The article presents the operational conjunction of the quality of life and supports paradigms in the new Quality of Life Supports Model (QOLSM) that integrates significant characteristics of the current transformation in the field of intellectual and developmental disabilities. These characteristics encompass a holistic and integrated approach, an approach focused on the human and legal rights of people with disabilities, eligibility for services and supports based on significant limitations in major areas of life activity, an emphasis on supports provided within inclusive community settings, and outcome assessment. The objectives of this article are to describe and indicate: (a) the four elements of the MOCA (fundamental values, individual and family quality of life domains, support systems and facilitating conditions); (b) how the MOCA can be used as a framework for the provision of supports, person-centered outcome assessment, organizational transformation and systems change; and (c) how MOCA is essential to the current paradigm shift in the field of Intellectual and Developmental Disabilities.

KeYwords: intellectual disability; developmental disabilities; quality of life; supports; model; paradigm; quality of life and supports model.

\section{Introducción general}

4 N LOS ÚlTimos 25 AÑos dos enfoques predominantes han coincidido en la manera de entender y atender a las personas con discapacidades intelectuales y del desarrollo (DID). Estas dos aproximaciones son el concepto de calidad de vida y el modelo de apoyos. El concepto de calidad de vida proporciona un importante marco conceptual para el desarrollo de las políticas sociales y para unas mejores prácticas profesionales, así como para la evaluación de resultados. Esto se debe a la naturaleza universal del concepto, los valores en los que se basa, el enfoque centrado en la persona y el énfasis en los resultados evaluados y referidos a la persona. El modelo de apoyos proporciona un marco de referencia para la planificación y ejecución de un conjunto coordinado de estrategias de apoyo a la persona que previenen o mitigan la discapacidad; promueven su desarrollo, educación e intereses, y mejoran su funcionamiento y bienestar personal.

Calidad de vida es un concepto global con evidencia científica de décadas, centrado en la persona, que proporciona información sobre lo que es importante en su vida y cuáles deben ser los resultados a conseguir, mientras que los apoyos se centran en cómo lograr esos resultados, son las estrategias y tácticas eficaces mediante las cuales se puede asegurar el éxito. Y teniendo claro el qué hacer y el cómo hacerlo sabemos cuál es el sentido de la navegación, y se ha de establecer una hoja de ruta centrada en las personas con DID y sus familias, para que los profesionales, organizaciones 
de apoyo y la implementación de la política pública avancen hasta poder evaluar los resultados de lo planificado.

Este trabajo, junto al que hemos publicado recientemente (Gómez et al., 2021; Schalock et al., 2020) y el de otros muchos autores, ha integrado esos dos caminos paralelos en un Modelo de Calidad de Vida y Apoyos [MOCA] que contribuye tanto teórica como operativamente al campo de las DID. Desde un punto de vista teórico, el modelo integra tres catalizadores críticos que provocan cambios positivos en la vida de las personas: las conexiones, las interacciones y las condiciones facilitadoras. Las conexiones se refieren a las que una persona o una familia tienen con otras personas, en las cuales las redes sociales y la tecnología brindan oportunidades para mejorar el bienestar y la calidad de vida. Las interacciones que resultan de estas conexiones proporcionan los sistemas de apoyo que facilitan el funcionamiento, los intereses y el bienestar personal. Las condiciones facilitadoras, que son básicas tanto para las conexiones como para las interacciones, se basan en el principio de desarrollo de oportunidades y en la provisión de apoyos basados en valores.

En las últimas décadas se ha cambiado positivamente la manera de entender a las personas con DID, y con ello la visión y orientación de las prácticas profesionales y organizacionales. La atención no se centra exclusivamente en el estudio y conocimiento de los defectos o limitaciones de la persona sino más bien en las características de su entorno. La clave de los avances en el bienestar personal y calidad de vida se relacionan con la gestión eficaz de los apoyos individuales, más allá de una rehabilitación o habilitación individual.

A pesar del gran cambio que se ha producido hacia modelos contextuales y sociales, todavía persisten y están presentes visiones simplistas que, sin mucho fundamento y con cierta visión de pensamiento mágico, apelan a la importancia del prefijo "neuro”, el cual aparece antes de las palabras educación, psicología, tecnología, marketing, etc. Esta neuro-moda, cuando pretende convertirse en explicación o en guía práctica, confunde y significa un retorno a enfoques del pasado científico, dando valor y examinando solamente aquello relacionado supuestamente con el cerebro. Y por ese camino no se encuentran soluciones. Todo esto teniendo en cuenta que la comprensión actual de la discapacidad intelectual parte de una aproximación integral e incluye los enfoques biomédicos, psicoeducativos, sociales y de la justicia (Schalock et al., 2016; Schalock, Luckasson et al., en prensa).

El Modelo de Calidad de Vida y Apoyos (MOCA) integra características significativas de la transformación actual en el campo de las DID. Estas características abarcan un enfoque holístico e integrado, centrado en los derechos humanos y legales de las personas con discapacidad, que basa sus decisiones sobre servicios y apoyos en las limitaciones significativas de las principales áreas de actividad de la vida, con un énfasis en los apoyos individualizados proporcionados dentro de ambientes inclusivos de la comunidad y promoviendo la evaluación de resultados. Adicionalmente, el modelo conceptual representa la conjunción operativa del subparadigma teórico de calidad de vida, que se basa en la investigación acumulada, y el subparadigma de apoyos como práctica profesional alternativa a los modelos tradicionales de rehabilitación (Schalock, et al., en prensa; Schalock y Verdugo, 2019). 
Los objetivos de este artículo son describir e indicar: (a) los cuatro elementos del MOCA: (a) valores fundamentales, dominios de calidad de vida individual y familiar, sistemas de apoyo y condiciones facilitadoras; (b) cómo se puede utilizar el MOCA como marco para la provisión de apoyos, la evaluación de resultados centrada en la persona, la transformación de la organización y el cambio de sistemas; y (c) cómo el MOCA es esencial para el cambio de paradigma actual en el campo de las DID.

\section{El Modelo de Calidad de Vida y Apoyos}

Un modelo conceptual debe facilitar la descripción y visualización de un fenómeno e incluir sus elementos críticos, así como indicar en qué manera esos elementos se pueden utilizar (Gómez et al., 2020). Como se muestra en la Figura 1, los cuatro elementos críticos del MOCA involucran los valores esenciales, las dimensiones de calidad de vida individual y familiar, los sistemas de apoyos y las condiciones facilitadoras.

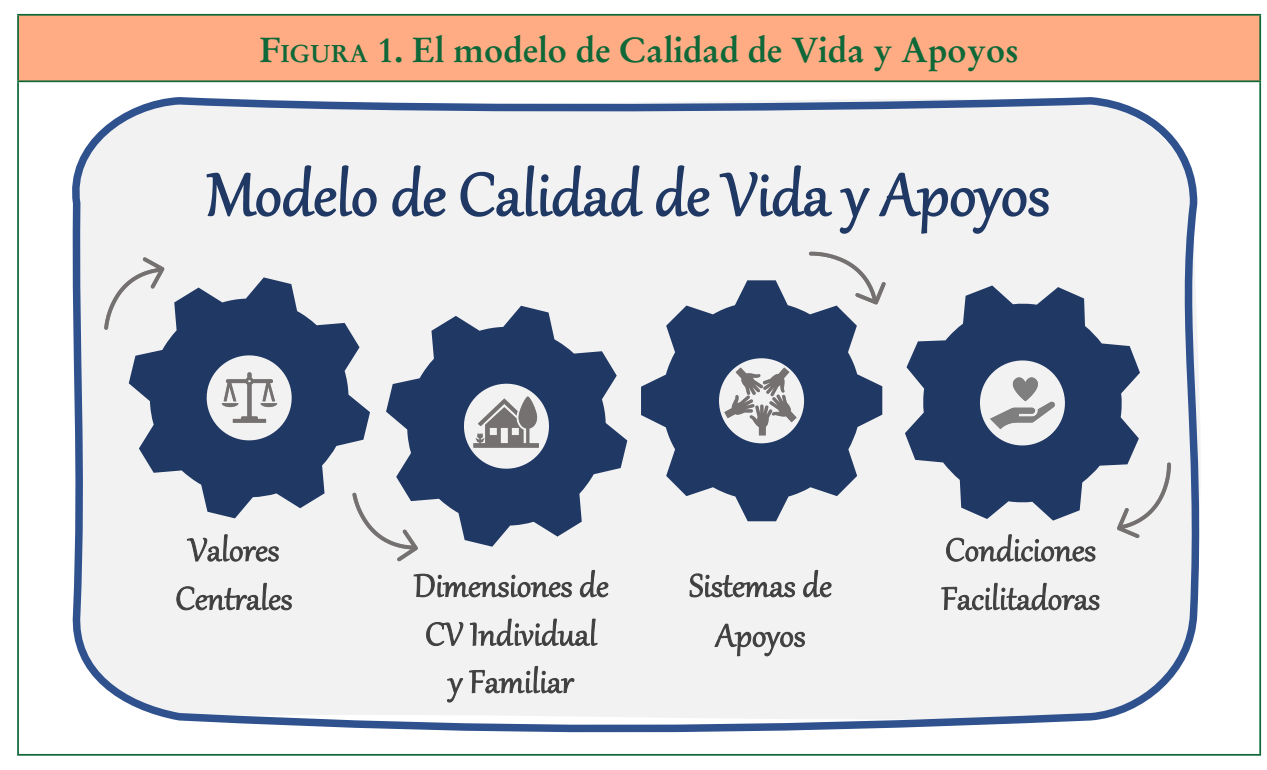

\subsection{Valores fundamentales}

Los valores fundamentales se derivan de las creencias y suposiciones que las personas tienen sobre las personas con DID y sobre su valor y potencial individual. Estos valores esenciales sirven para crear expectativas y guían las políticas y prácticas con respecto a las personas con DID y el papel que desempeñan en la sociedad. Cualquier 
proceso de cambio o transformación de una organización hacia modelos más eficaces debe comenzar con información y capacitación sobre estos valores fundamentales. Sin modificar los modelos mentales difícilmente se pueden generar distintas metas y acciones e implementar un enfoque positivo sobre las personas.

A continuación, se enumeran los principales valores incorporados en el MOCA, con algunas de sus mejores referencias ilustrativas:

- Los derechos humanos y legales de las personas (Claes et al., 2016; Harpur, 2012; Gómez et al., 2020; Mittler, 2015; Navas et al., 2012; Verdugo et al., 2012).

- La capacidad y el potencial de las personas para crecer y desarrollarse (Nussbaum, 2011; Wehmeyer, 2013).

- El énfasis en la autodeterminación (Lachapelle et al., 2005; Schalock et al., 2016; Shogren et al., 2017; Vicente et al., 2018, 2020).

- La naturaleza universal de la multidimensionalidad de la calidad de vida y su énfasis en la inclusión y la equidad para las personas con discapacidad y sus familias (Aza et al., 2020; Consortium on Quality of Life, 2019; Fernández et al., 2019; Isaacs et al., 2007; Morán et al., 2019; Schalock y Keith, 2016; Schalock y Verdugo, 2002, 2012).

- El compromiso de abordar las necesidades de apoyo de una persona y fomentar oportunidades para mejorar su funcionamiento individual y bienestar personal (Buntinx et al., 2018; Onken, 2018; Qian et al., 2019).

\subsection{Dimensiones de calidad de vida individual y familiar}

Los trabajos pioneros de Brown (1993), Goode (1994), Felce (1997), Keith et al. (1996), Parmenter (1992), Raphael et al. (1996) y Schalock y Verdugo (2002) condujeron al desarrollo y publicación (Schalock et al., 2002, 2011) de un documento de consenso sobre los principios subyacentes a la conceptualización, medición y aplicación del concepto de calidad de vida que a continuación se exponen:

- Principios de conceptualización: la calidad de vida es multidimensional y está influenciada por factores personales y ambientales y su interacción; tiene las mismas dimensiones para todas las personas; tiene componentes subjetivos y objetivos; y se ve favorecida por la autodeterminación, los recursos, el propósito de la vida y el sentido de pertenencia.

- Principios de medición: la medición en la calidad de vida implica el grado en que las personas tienen experiencias de vida que valoran; refleja los dominios que contribuyen a una vida plena e interconectada; considera los contextos de los entornos físicos, sociales y culturales que son importantes para las personas; e incluye medidas de experiencias tanto comunes como únicas de las personas.

- Principios de aplicación: la aplicación del concepto de calidad de vida sirve para mejorar el bienestar personal con respecto al contexto cultural de cada uno, debe basarse en evidencias y los principios de calidad de vida deben ser la base de las intervenciones y los apoyos, así como ocupar un lugar destacado en la educación y la formación de los profesionales. 
A partir de las aportaciones de los autores relacionados y de los principios propuestos se desarrollaron diferentes modelos conceptuales que se centraron en la calidad de vida referida a individuos o a familias. Las dimensiones de calidad de vida que abarcan los modelos comúnmente referenciados en las publicaciones científicas son muy similares (Gómez et al., 2011; Gómez et al., 2020). En la Tabla 1, se enumeran los dominios de calidad de vida individuales (Schalock y Verdugo, 2002, 2012) y familiares (Brown et al., 2006.; Isaacs et al., 2007; Summers et al., 2005; Zuna et al., 2010) habitualmente propuestos. Las dimensiones reflejan la propiedad universal del concepto de calidad de vida, un enfoque centrado en el individuo o en la familia, y los principios de aplicación relacionados con la equidad, la inclusión, la autodeterminación, el empoderamiento y la evaluación de resultados. Las dimensiones también proporcionan un marco para usar el MOCA para la provisión de apoyos, la evaluación de resultados centrada en la persona, la transformación de la organización y el cambio de sistemas.

\begin{tabular}{|l|l|}
\hline \multicolumn{2}{|c|}{ TABLA 1. Dimensiones de Calidad de vida individual y familiar } \\
\hline Dimensiones individuales referidas a la CDV & Dimensiones familiares referidas a la CDV \\
\hline Desarrollo Personal & Rol Parental \\
\hline Autodeterminación & Interacciones Familiares \\
\hline Relaciones Interpersonales & Bienestar Emocional \\
\hline Inclusión Social & Bienestar Físico \\
\hline Derechos & Apoyos Relacionados con la Discapacidad \\
\hline Bienestar Emocional & Apoyo de Otras Personas \\
\hline Bienestar Físico & Desarrollo Profesional \\
\hline Bienestar Material & Interacción Comunitaria \\
\hline
\end{tabular}

\subsection{Sistemas de apoyos}

Los sistemas de apoyos son un componente clave del modelo aquí propuesto. Los modelos de apoyo, como el MOCA, se centran en la adecuación entre las personas y sus entornos, y abordan la discapacidad como una expresión de las limitaciones en el funcionamiento dentro de un contexto social. El MOCA postula además que: (a) la discapacidad no es fija ni dicotómica, sino flexible, según las fortalezas y limitaciones de la persona o la familia y los apoyos disponibles en el entorno; y (b) se pueden mitigar los efectos de la propia discapacidad diseñando intervenciones, servicios y apoyos basados en la participación colaborativa y una comprensión de la discapacidad que proviene de la experiencia y el conocimiento vividos (Schalock et al., 2020; Thompson et al., 2014).

Desde la introducción del concepto de apoyos en 1992, en el manual de la entonces denominada Asociación Americana sobre Retraso Mental [AAMR] (Luckasson et al.) y en sus revisiones posteriores como Asociación Americana sobre Discapacidades Intelectuales y del Desarrollo [AAIDD] (Luckasson et al., 1992, 2002; Schalock 
et al., 2010, 2021), el concepto de apoyos y su implementación ha tenido un impacto determinante en el campo profesional y científico de las DID con: (a) el uso de escalas estandarizadas de evaluación de las necesidades de apoyo (por ejemplo, Aguayo et al., 2019; Stancliffe et al., 2016; Thompson et al., 2015, 2016; Verdugo et al., 2020, 2021); (b) el desarrollo de estándares de apoyo (por ejemplo, Amor et al., 2021; Buntinx et al., 2018); (c) la implementación de planes de apoyo personal que alinean las necesidades de apoyo, los objetivos personales, las estrategias de apoyo y los resultados valorados por una persona (por ejemplo, Schalock et al., 2018); y (d) la implementación de equipos de apoyo estructurados horizontalmente que desarrollan planes individuales fáciles de usar (por ejemplo, Amor et al., 2020; Reinders y Schalock, 2014; SánchezGómez et al., 2020; Verdugo et al., 2020).

Los sistemas de apoyos son una amplia gama de recursos y estrategias que previenen o mitigan una discapacidad o sus efectos; promueven el desarrollo, la educación, los intereses y el bienestar de las personas con DID o de sus familias; y mejoran el funcionamiento y el bienestar individual o familiar. Una agrupación comúnmente utilizada de los elementos de los sistemas de apoyos engloba (Schalock et al., 2020):

- Elección y autonomía personal, que implica tener oportunidades para tomar decisiones y ejercer la autodeterminación, ser reconocido como persona ante la ley y disfrutar de capacidad jurídica en igualdad de condiciones con quienes no tienen discapacidad. La elección y la autonomía personal se facilitan mediante apoyos en la toma de decisiones.

- Los entornos inclusivos son aquellos que brindan acceso a recursos, información y relaciones; fomentan el crecimiento y el desarrollo y apoyan a las personas, y se adaptan a las necesidades psicológicas de autonomía, competencia y afinidad.

- Los apoyos genéricos son aquellos que están ampliamente disponibles para la población en general, incluidos los apoyos naturales, tecnología, prótesis, oportunidades de aprendizaje de por vida, adaptaciones razonables, dignidad y respeto, y fortalezas o activos personales.

- Los apoyos especializados son intervenciones, estrategias y terapias profesionales.

Como elemento clave del MOCA, los sistemas de apoyos brindan un marco de referencia para mejorar el funcionamiento y el bienestar individual o familiar. Para este fin, se utilizan en la planificación de apoyos una amplia gama de recursos y estrategias, y también sirve como guía de los esfuerzos colectivos, descritos en la siguiente sección, con respecto a la provisión de apoyos, la evaluación de resultados centrada en la persona, la transformación de la organización y el cambio de sistemas.

\subsection{Condiciones facilitadoras}

Las condiciones facilitadoras son factores contextuales operativos que influyen en la aplicación con éxito del MOCA. Estos factores contextuales están influenciados por -e interactúan con- propiedades del micro-, meso- y macrosistema (Shogren et 
al., 2020, en prensa). Se puede encontrar información adicional sobre la influencia de estas condiciones facilitadoras en los componentes del MOCA en el trabajo de Buntinx et al. (2018), Onken (2018), Qian et al. (2019), Shogren et al. (2020) y el Consortium on Quality of Life (2019).

- Las condiciones que facilitan la calidad de vida incluyen: participación en la comunidad, promoción del sentido de pertenencia, maximización de capacidades y oportunidades, libertad para participar en las principales actividades de la vida, entornos seguros y protegidos, y un compromiso con las metas que son importantes para la persona o familia.

- Las condiciones que facilitan el apoyo incluyen: comprender las necesidades de apoyo de la persona; el compromiso de abordar las necesidades de apoyo de la persona y mejorar sus metas personales; la disponibilidad y accesibilidad de los apoyos; el conocimiento de los elementos de los sistemas de apoyo; proveedores de apoyo competentes y con experiencia, consistencia y estabilidad en la prestación de apoyos, y coordinación y gestión de apoyo apropiadas.

\section{Utilización del Modelo de Calidad de Vida y Apoyos (MOCA)}

La integración del concepto de calidad de vida y el modelo de apoyos en el MOCA proporciona un marco teórico y profesionalmente sólido para la provisión de apoyos, evaluación de resultados centrada en la persona, transformación de las organizaciones y cambio de sistemas. Cada uno de estos usos se describe en esta sección del artículo. Estos cuatro usos reflejan cómo el MOCA puede ser utilizado para: (a) alinear las necesidades de apoyo de una persona con estrategias de apoyo individualizadas y resultados significativos; (b) conectar prácticas profesionales individuales con las prioridades y misiones de las organizaciones; (c) sincronizar las políticas y toma de decisiones entre las organizaciones y el sistema; (d) desarrollar un esquema conceptual basado en valores para las políticas de discapacidad; y (e) establecer parámetros para la evaluación centrada en la persona.

\subsection{Prestación de apoyos}

Las familias, los cuidadores primarios, el personal de apoyo y los maestros son los principales proveedores de apoyo en todo el mundo. Las tres estrategias más aplicables en todos los programas de prestación de apoyos son: (a) un énfasis en la calidad de vida, (b) la provisión de apoyos relacionados con la elección y la autonomía personal y (c) la provisión de apoyos genéricos disponibles para cualquier persona y que pueden ser proporcionados por distintos proveedores. Estas tres estrategias proporcionan conexiones, interacciones y condiciones facilitadoras.

Énfasis en la calidad de vida. Los principios y valores de calidad de vida relacionados con la dignidad, equidad, inclusión, autodeterminación y el empoderamiento reflejan el papel clave que desempeña el énfasis en la calidad de vida en la vida de 
las personas. La incorporación de los valores y principios de calidad de vida en las interacciones con la persona asegura que los proveedores de apoyo se den cuenta de que la calidad de vida de uno, independientemente del alcance de sus limitaciones, se compone de muchas dimensiones que reflejan el bienestar personal; que las áreas de calidad de vida son las mismas para todas las personas, aunque esas áreas pueden valorarse de manera diferente por cada uno; que la calidad de vida de uno tiene aspectos subjetivos y objetivos; y, finalmente, que la calidad de vida de cada uno es dinámica y está sujeta a cambios. Hay que resaltar que el énfasis en la calidad de vida incorpora un enfoque holístico de la persona, una visión global que permite a las organizaciones de apoyo "pensar más allá de la discapacidad de la persona” y optimizar las condiciones asociadas con oportunidades basadas en principios y apoyos basados en valores.

Énfasis en la elección y la autonomía personal. Unos elementos clave de los sistemas de apoyo son la elección y la autonomía personal pues tienen la capacidad de mitigar las DID; promover el desarrollo, la educación y los intereses de una persona; y mejorar el funcionamiento y el bienestar del individuo y de la familia. El ejercicio de la propia elección y la autonomía personal aumenta no solo la motivación y la satisfacción de las necesidades psicológicas relacionadas con la independencia, la afinidad y la competencia, sino que también disminuye la conducta inadaptada (Deci y Ryan, 2012). La elección y la autonomía personal también se enfatizan en el artículo 12 de la Convención de Derechos de Naciones Unidas (CDPD; Naciones Unidas, 2006), que reconoce los derechos de las personas con discapacidad a la capacidad jurídica. Como expusieron Glen (2015) y Luckasson et al. (2017), todas las personas, incluidas aquellas con DID, tienen derecho a contar con opciones, tomar sus propias decisiones, y que esas opciones y decisiones sean reconocidas legalmente.

Uso de apoyos genéricos. Los apoyos genéricos incluyen aquellas estrategias de apoyo que están disponibles para cualquier persona, con o sin discapacidad, y que pueden ser proporcionadas por muchos proveedores de apoyo. La inclusión de apoyos genéricos como un elemento de los sistemas de apoyos permite a los proveedores de apoyos ampliar su enfoque desde el uso exclusivo de intervenciones profesionales y apoyos pagados hasta la provisión de apoyos genéricos que los miembros de la familia, los cuidadores primarios, los proveedores de apoyo informal, los maestros o las propias personas pueden brindar. Los apoyos genéricos incluyen apoyos naturales, tecnología, prótesis, educación a lo largo de la vida, acomodaciones razonables, dignidad y respeto, y fortalezas y activos personales (Consortium on Quality of Life, 2019; Lombardi et al., 2020, Schalock et al., 2019; Stancliffe et al., 2016; Thompson et al., 2009, 2014).

\subsection{Evaluación de resultados centrada en la persona}

Tanto el concepto de calidad de vida como el modelo de apoyos están centrados en la persona. El MOCA proporciona un marco de referencia para la evaluación de resultados centrados en la persona, que es el esfuerzo sistemático que alinea los valores fundamentales con una comprensión moderna de las DID, los apoyos individua- 
lizados y los resultados e impactos significativos. Este enfoque de la evaluación de resultados implica un acuerdo o alianza de colaboración entre una persona, una organización o sistema de servicios humanos y un equipo. El propósito de la evaluación centrada en la persona es emplear el conocimiento, las habilidades y los recursos de la alianza establecida para medir y utilizar eficazmente la información de los resultados para mejorar el bienestar personal, aumentar la transparencia, facilitar la rendición de cuentas y ampliar la comprensión de las personas (Schalock y Luckasson, 2020).

Se puede utilizar un modelo lógico para visualizar e implementar un enfoque de evaluación centrado en la persona. Como describen Schalock y Luckasson (2020), los cuatro componentes de un modelo de evaluación de resultados centrado en la persona implican las siguientes fases: input, proceso, resultados y productos.

- El componente de entrada o input abarca valores fundamentales (como los enumerados anteriormente), además de un enfoque funcional y un enfoque holístico para las DID.

- El componente de proceso (o rendimiento) involucra los sistemas de apoyo que se utilizan para incorporar los valores centrales en el marco de evaluación, maximizar la calidad de vida y apoyar las condiciones que facilitan y mejoran el bienestar personal y los resultados de calidad de vida.

- El componente de resultado se centra en los resultados seleccionados y medidos de acuerdo con las dimensiones de calidad de vida referidas a individuos y familias.

- El componente de producto enfatiza los impactos significativos de la evaluación centrada en la persona para mejorar el bienestar personal, aumentar la transparencia, facilitar la rendición de cuentas y ampliar la comprensión.

Un enfoque centrado en la persona para la evaluación de resultados involucra un enfoque funcional y holístico de las DID, el modelo socioecológico de la discapacidad, un sistema de prestación de servicios o apoyos basado en los apoyos, prácticas basadas en evidencia y un énfasis en resultados significativos (Schalock et al., 2020).

\subsection{Transformación de la organización}

Los procesos de innovación y cambio deben ser implementados por cada organización partiendo de un análisis particular de su contexto en los tres niveles del sistema (Schalock y Verdugo, 2012, 2013), sin olvidar que lo primero de todo es informar, formar e involucrar en el proceso a todo el personal implicado. A partir de ese análisis contextual, las organizaciones han de desarrollar sus actividades para ofrecer más oportunidades de avanzar en el ejercicio de derechos y mejora del bienestar individual de los usuarios de sus servicios y de sus familias.

Las organizaciones que han implementado uno o más componentes del MOCA han cambiado sus políticas y prácticas y, por lo tanto, se han transformado de manera significativa. Un ejemplo de transformación consiste en implementar o en mejorar los sistemas de apoyos. Otros ejemplos son alinear las necesidades de apoyo de una 
persona con estrategias específicas de apoyo para obtener resultados significativos; conectar las prácticas profesionales sobre la persona o su familia con las prioridades y misiones de la organización; alinear la calidad de vida con las estrategias de apoyo y la toma de decisiones entre el nivel de la organización y el de sistemas; desarrollar un marco de referencia para políticas y prácticas basado en el MOCA, y realizar una evaluación de resultados centrada en la calidad de vida (Amor et al., 2020; Baker et al., 2016; Schalock y Keith, 2016; Schalock y Verdugo, 2013; Thompson et al., 2014).

Las organizaciones se transforman cuando desarrollan nuevas formas de pensar e implementan nuevas políticas y prácticas relacionadas con su sistema de prestación de servicios. El MOCA puede guiar la aplicación de un Sistema de Prestación de Calidad de Vida y Apoyos (Reinders y Schalock, 2014). Dicho sistema puede visualizarse y facilitarse mediante el uso de un modelo lógico cuyos componentes de entrada, proceso y resultado o producto se muestran en la Figura 2.

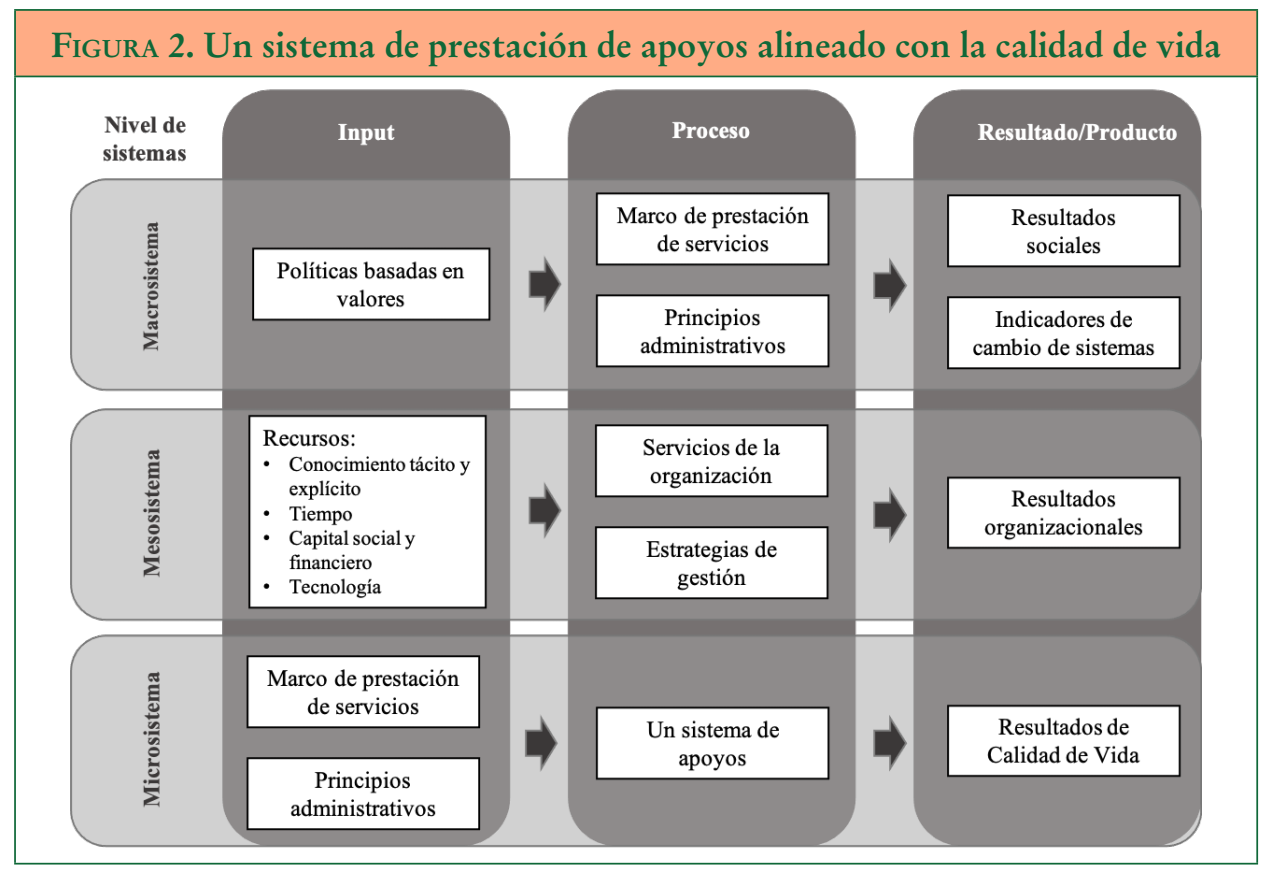

La Figura 2 muestra también cómo los componentes de entrada, proceso y resultado o producto del sistema se pueden analizar y alinear tanto vertical como horizontalmente. La alineación horizontal ubica los componentes de prestación de apoyos en una secuencia lógica para la planificación, implementación, supervisión y evaluación. La alineación vertical asegura que en el "input” las políticas basadas en valores del macrosistema están alineadas con los recursos de la organización, el marco de presta- 
ción de servicios y los principios administrativos. En el “proceso”, la alineación vertical garantiza que el marco de prestación de servicios y los principios administrativos están alineados con los servicios y las estrategias de gestión de las organizaciones, y que se brindan apoyos individualizados. En el "resultado o producto", la alineación vertical asegura que los resultados o productos asociados con la provisión de apoyo, la transformación de la organización y el cambio de sistemas están relacionados conceptual y operativamente con los componentes del modelo.

\subsection{Cambio de sistemas}

El cambio de sistemas hay que entenderlo como un proceso intencional diseñado para cambiar los componentes y estructuras habituales de un sistema, las cuales se consideran obsoletas o poco eficaces. Hay muchas formas de cambiar los sistemas de funcionamiento en los que participamos, pero siempre es un proceso complejo que requiere abordarse en varias fases y con una alianza entre distintas partes del sistema. Se pueden destacar como principios esenciales del cambio: la comprensión de las necesidades de cambio y de los recursos disponibles, la involucración de varios actores, definir un esquema del sistema y del cambio a lograr, implementarlo conjuntamente, apoyarse y promover liderazgos diversos y generar una cultura de aprendizaje. Para información detallada sobre las estrategias de cambio organizacional y de los sistemas conviene consultar el libro El cambio en las organizaciones de discapacidad. Estrategias para superar sus retos y hacerlo realidad (Schalock y Verdugo, 2012) y las aportaciones de Baker et al. (2016), Schalock y Verdugo (2013) y Schalock et al. (2018).

Por otro lado, la CDPD constituye una oportunidad única para mejorar el bienestar personal de las personas con discapacidad, ya que sus artículos incorporan los principios y valores incluidos en el concepto de calidad de vida, y sus objetivos alientan a los signatarios para realizar ajustes razonables en sus sistemas de prestación de apoyos de manera que permitan a las personas con discapacidad ejercer sus derechos (Mittler, 2015). La implementación de cambios en los sistemas es un desafío importante para todos los países que firmaron la CDPD y, por tanto, para todas las comunidades autónomas, porque deben afrontar el reto de hacer “ajustes razonables" para permitir que las personas con discapacidades y sus familias avancen en sus derechos y experimenten una mejor calidad de vida. El cambio de sistemas que supone la Convención se puede y es muy oportuno basarlo en la alineación de las dimensiones de calidad de vida con los artículos de aquella y con los elementos de los sistemas de apoyo. Y eso es lo que han demostrado recientemente algunas investigaciones (Claes et al., 2016; Gómez et al., 2020b; Lombardi et al., 2019, 2020; Verdugo et al., 2012).

El alineamiento de la CDPD con las dimensiones de calidad de vida y las estrategias de apoyo asociadas proporciona un marco de referencia basado en la evidencia para implementar aquella. Específicamente, el planteamiento se puede usar para guiar los esfuerzos a nivel de sistemas para materializar los objetivos de la CDPD en políticas y prácticas viables para las personas con discapacidad; para utilizar las dimensiones de calidad de vida en la provisión de apoyos y evaluación de resultados deseados, 
y también para desarrollar prácticas basadas en la organización alineadas con artículos específicos de la Convención.

El progreso realizado recientemente con respecto al uso de un marco de referencia de calidad de vida basado en la evidencia para la implementación de la CDPD ha sido sintetizado por Gómez, Monsalve et al. (2020). La publicación incluye un resumen de los modelos conceptuales de calidad de vida utilizados para supervisar la CDPD, los instrumentos utilizados para evaluar los derechos propuestos y los resultados personales asociados con derechos específicos de la Convención. Y se ha iniciado el camino de construir y validar un instrumento para evaluar los resultados de personas con DID en las ocho dimensiones de calidad de vida en relación con los artículos de la CDPD.

\section{El MOCA y el Paradigma Calidad de Vida y Apoyos emergente}

Un paradigma es el conjunto de creencias, hipótesis, políticas y prácticas compartidas por muchas personas sobre un ámbito de conocimiento específico que sirve para guiar los esfuerzos en resolver problemas y desarrollar nuevos conocimientos (Thompson et al., 2014). El campo de las DID está experimentando actualmente un “cambio de paradigma” en este conjunto de creencias, supuestos, políticas y prácticas. Este paradigma emergente, al que nos referimos como Paradigma de Calidad de Vida y Apoyos (PCVA), reemplaza el paradigma histórico que enfatizaba la defectología, la segregación, la devaluación de la persona y los servicios basados en instituciones con un enfoque comunitario basado en el concepto de calidad de vida y apoyos individualizados (Gómez et al., 2021; Schalock et al., 2020).

El MOCA es fundamental para el PCVA emergente. Siguiendo a Gómez et al. (2021), el PCVA se caracteriza por estar basado en la teoría y en un modelo conceptual validado como es el MOCA. Como se muestra en la Figura 1, los elementos críticos de este modelo involucran valores centrales, dimensiones de calidad de vida referidas a personas y familias, sistemas de apoyo y condiciones facilitadoras. Además, el PCVA es:

- Ético y engloba valores fundamentales. Estos valores involucran la valía y autonomía individual, la capacidad de las personas para crecer y desarrollarse, la naturaleza universal de la multidimensionalidad de la calidad de vida, el énfasis en la inclusión y la equidad, el compromiso de abordar las necesidades de apoyo de una persona y el fomento de oportunidades para mejorar el funcionamiento y el bienestar personal.

- Flexible y utilizado para múltiples propósitos. Estos propósitos, ya descritos antes, incluyen la provisión de apoyos, la evaluación de resultados centrada en la persona, la transformación de la organización y el cambio de sistemas.

- Adaptable y proporciona un marco de referencia para adaptarse a las condiciones facilitadoras basadas en el contexto.

- Medible y evaluable. Aunque más allá del alcance de este artículo, la influencia y el impacto del PCVA emergente puede evaluarse mediante el uso de una o 
más estrategias de evaluación: centradas en principios, centradas en la utilización, centradas en resultados y centradas en procesos (Gómez et al., 2021; Schalock et al., 2020).

\section{Conclusión}

El concepto de calidad de vida y el modelo de apoyos han tenido un impacto significativo por sí solos en el campo de las DID durante los últimos 25 años. Al integrar ambos enfoques en el MOCA descrito en este artículo, entendemos que las organizaciones y profesionales, junto a responsables de gestión en las administraciones, tienen ahora un catalizador aún más poderoso para lograr cambios positivos en las políticas y prácticas que mejoran la calidad de vida de las personas con DID y sus familias.

La conjunción de los subparadigmas de calidad de vida y apoyos en un solo modelo orienta, refuerza y estimula los procesos de cambio iniciados por muchas organizaciones y la planificación de otros nuevos procesos. Hay que reconocer que este planteamiento conjunto es el resultado de la visión e iniciativas de investigadores, organizaciones y profesionales que realizaron el arduo y dedicado trabajo involucrado en la conceptualización y medición, la transformación de la organización y el cambio de sistemas. Todo ello, con la colaboración imprescindible y el compromiso y esfuerzos de las personas con DID y sus familias para defender los valores y avanzar en oportunidades, políticas y prácticas esenciales que se basan en los apoyos y se centran en la calidad de vida.

La situación derivada de la pandemia mundial desde el año 2019 es una oportunidad para el cambio, innovación y mejora de las organizaciones y los servicios habitualmente desarrollados. Las consecuencias de la COVID-19 han dejado muy claro que las grandes residencias y los enfoques segregados llevan a resultados muy negativos, con consecuencias incluso de muerte y enfermedad más comunes que en otras alternativas. Las administraciones y las organizaciones son las responsables de impulsar los procesos de cambio necesarios para abandonar enfoques pasivos, despersonalizados y no centrados en la persona. La emergencia y generalización de enfoques más comunitarios centrados en la calidad de vida y en los apoyos es la respuesta apropiada, tal como nos indican los resultados de investigaciones recientes (Navas et al., 2020; Navas et al., 2020). Los derechos individuales que proclama la CDPD de Naciones Unidas tienen su sentido natural en espacios de libertad y oportunidades. La segregación es una negación de derechos.

\section{Referencias bibliográficas}

Aguayo, V., Verdugo, M. Á., Arias, V. B., Guillén, V. M. y Amor, A. M. (2019). Assessing support needs in children with intellectual disability and motor impairments: measurement invariance and group differences. Journal of Intellectual Disability Research, 63(12), 14131427. https://doi.org/10.1111/jir.12683 
EL MODELO DE CALIDAD DE VIDA Y APOYOS:

LA UNIÓN TRAS VEINTICINCO AÑOS DE CAMINOS PARALELOS

M. Á. VERDUGO, R. L SCHALOCK Y L. E. GÓMEZ

Amor, A. M., Fernández, M., Verdugo, M. Á., Aza, A. y Schalock, R. L. (2020). Shaping the faces of the prism: rights, supports, and quality of life for enhancing inclusive education opportunities in students with intellectual disability. Cztowiek-NiepetnosprawnośćSpoteczeństwo, 49, 5-33.

Amor, A. M., Verdugo, M. Á., Arias, B., Fernández, M. y AzA, A. (2021). Examining the suitability of the list of indicators describing age-related typical support needs. International Journal of Environmental Research and Public Health, 18, 764. https://doi.org/10.3390/ ijerph18020764

Aza, A., Verdugo, M. Á., Orgaz, M. B., Fernández, M. y Amor, A. M. (2020). Adaptation and validation of the self-report version of the scale for measuring quality of life in people with acquired brain injury (CAVIDACE). Quality of Life Research, 29(4), 1107-1121. https:// doi.org/10.1007/s11136-019-02386-4

Baker, A., Salisbury, B. y Collins, D. (2016). Changing service delivery systems: an example from Community Living British Columbia. En R. L. Schalock y K. D. Keith (Eds.), Cross-cultural quality of life: enhancing the lives of people with intellectual disability (pp. 149-166). American Association on Intellectual and Developmental Disabilities.

Brown, I., Brown, R., Baum, N. T., Isaacs, B. J., Myerscough, T., Neikrug, S., Roth, D., SHEARER, J. y WANG, M. (2006). Family quality of life survey: main caregivers of people with intellectual or developmental disabilities. Surrey Place Centre.

Brown, R. I. (1993). Quality of life issues in aging and intellectual disability. Australia and New Zealand Journal of Developmental Disabilities, 18, 219-227.

Buntinx, W. H. E., Tu Tan, I. y Aldenkamp, A. P. (2018). Support values through the eyes of the patient: an exploratory study into long-term support for persons with refractory epilepsy. Epilepsy and Behavior, 82, 155-163. https://doi.org/10.1016/j.yebeh.2018.02.031

Claes, C., Vandenbussche, H. y Lombardi, L. (2016). Human rights and quality of life domains: identifying cross-cultural indicators. En R. L. Schalock y K. D. KeITH (Eds.), Crosscultural quality of life: enhancing the lives of people with intellectual and developmental disabilities ( $\left.2^{\text {nd }} \mathrm{Ed}\right)$ (pp. 167-174). American Association on Intellectual and Developmental Disabilities.

CONSORTIUM ON QuAlity OF Life. (2019). Towards a consensus document regarding the conceptualization, measurement, and application of the quality life concept. University of Gent.

Deci, E. L. y Ryan, R. M. (2012). Motivation, personality, and development within embedded social contexts: an overview of self-determination theory. En R. M. RyAN (Ed.), The Oxford handbook of human motivation (pp. 85-107). Oxford University Press.

Felce, D. (1997). Defining and applying the concept of quality of life. Journal of Intellectual Disability Research, 41, 126-135. https://doi.org/10.1111/j.1365-2788.1997.tb00689.x

Fernández, M., Gómez, L. E., Arias, V. B., Aguayo, V., Amor, A. M., Andelic, N. y VerduGO, M. Á. (2019). A new scale for measuring quality of life in acquired brain injury. Quality of Life Research, 28(3), 801-814.

GLEN, K. B. (2015). Supported decision making and the human rights of legal capacity. Inclusion, 3, 2-16. https://doi.org/ 10.1352/2326-6988-3.1.2

Gómez, L. E., Monsalve, A., Moran, L., Alcedo, M. A., Lombardi, M. y Schalock, R. L. (2020). Measurable indicators of CRPD for people with intellectual and developmental disabilities within the framework of quality of life. International Journal of Environmental Research and Public Health, 17, Article 5123. https://doi.org/10.3390/ijerph17145123

Gómez, L. E., Schalock, R. L. y Verdugo, M. Á. (2020). The role of moderators and mediators in implementing and evaluating intellectual and developmental disabilities- rela-

Ediciones Universidad de Salamanca / CC BY-NC-ND

Siglo Cero, vol. 52 (3), 2021, julio-septiembre, pp. 9-28 
EL MODELO DE CALIDAD DE VIDA Y APOYOS:

LA UNIÓN TRAS VEINTICINCO AÑOS DE CAMINOS PARALELOS

M. Á. VERDUGO, R. L SCHALOCK Y L. E. GÓMEZ

ted policies and practices. Journal of Developmental and Physical Disabilities, 32, 375-393. https://doi.org/10.1007/s10882-019-097202-3

Gómez, L. E., Schalock, R. L. y Verdugo, M. Á. (2021). A new paradigm in the field of intellectual and developmental disabilities: characteristics and evaluation. Psicothema, 33(1), 28-35. https://doi.org/10.7334/psicothema2020.385

Gómez, L. E., Verdugo, M. Á., Arias, B. y Arias, V. B. (2011). A comparison of alternative models of individual quality of life for social service recipients. Social Indicators Research, 101, 109-126. https://doi.org/ 10.1007/s11205-010-9639-y

Goode, D. (Ed.). (1994). Quality of life for persons with disabilities: international issues and perspectives. Brookline Press.

HARPuR, P. (2012). Embracing the new disability rights paradigm: the importance of the Convention on the Rights of Persons with Disabilities. Disability and Society, 27, 1-14. https:// doi.org/ 10.1080/09687599.2012.631794

Isaacs, B. J., Brown, I., Brown, R. I., Baum, N., Myserscough, T., Neikrug, A., Roth, D., SHEARER, J. y WANG, M. (2007). The International Family Quality of Life Project. Journal of Policy and Practice in Intellectual Disabilities, 4, 177-185. https://doi.org/ 10.1111/j.17411130.2007.00116.x

Keith, K. D., HeAL, L. y Schalock, R. L. (1996). Cross-cultural measurement of critical quality of life concepts. Journal of Intellectual and Developmental Disabilities, 21, 273-293. https://doi.org/10.1080/13668259600033201

Lachapelle, Y., Wehmeyer, M. L., Haelewyck, M. C., Courbois, Y., Keith, K. D., SchaLoCK, R. L., Verdugo, M. Á. y Walsh, P. N. (2005). The relationship between quality of life and self-determination: an international study. Journal of Intellectual Disability Research, 49(10), 740-744. https://doi:10.1111/j.1365-2788.2005.00743.x

Lombardi, M., Chu, C., Claes, C. y Schalock, R. L. (2020). Towards an international definition of supports, systems of supports, and elements of systems of supports. Manuscrito enviado para publicación.

Lombardi, M., Vandenbussche, H., Claes, C., Schalock, R. L., De Maeyer, J., y VandeVELDE, S. (2019). The concept of quality of life as a framework for implementing the UNCRPD. Journal of Policy and Practice in Intellectual Disabilities, 16(3), 180-190. https://doi. org/10.1111/jppi.12279

Luckasson, R., Borthwick-Duffy, S. A., Buntinx, W. H. E., Coulter, D., Craig, E. M., Reeve, A., Schalock, R. L., Snell, M. E., Spitalnik, D. M., Spreat, S. y Tassé, M. J. (2002). Mental retardation. Definition, classification and systems of supports (10th Ed). American Association on Mental Retardation. [Traducción de M. Á. Verdugo y C. JenARo. Retraso mental: Definición, clasificación y sistemas de apoyo (10. a edición). American Association on Mental Retardation/Alianza Editorial, 2004].

Luckasson, R., Coulter, D. L., Polloway, E. A., Reiss, S., Schalock, R. L., Snell, M. E., Spitalnik, D. M. y Stark, J. A. (1992). Mental retardation. Definition, classification and systems of supports (10th Ed). American Association on Mental Retardation. [Traducción de M. Á. Verdugo y C. Jenaro. Retraso mental: definición, clasificación y sistemas de apoyo (9. a edición). American Association on Mental Retardation/Alianza Editorial, 1997].

Luckasson, R., Coulter, D. L., Polloway, E. A., Reiss, S., Schalock, R. L., Snell, M. E., Spitalnik, D. M. y StARK, J. A. (2002). Mental retardation. Definition, classification and systems of supports (10th Ed). American Association on Mental Retardation. [Traducción de M. Á. Verdugo y C. JenARO. Retraso mental: definición, clasificación y sistemas de apoyo (10. a edición). American Association on Mental Retardation/Alianza Editorial, 2004].

Ediciones Universidad de Salamanca / CC BY-NC-ND

Siglo Cero, vol. 52 (3), 2021, julio-septiembre, pp. 9-28 
EL MODELO DE CALIDAD DE VIDA Y APOYOS:

LA UNIÓN TRAS VEINTICINCO AÑOS DE CAMINOS PARALELOS

M. Á. VERDUGO, R. L SCHALOCK Y L. E. GÓMEZ

Luckasson, R., Ford, M. E., Mcmillan, E. D., Misilo, F. M. Jr. y Nygren, M. A. (2017). Intellectual disability policy as developed, expressed, and evaluated in AAIDD/The Arc joint statements. Intellectual and Developmental Disabilities, 55, 269-275. https://doi.org/ 10.1352/1934-9556-55.4.269

MitTler, P. (2015). The UN Convention on the Rights of Persons with Disabilities: implementing a paradigm shift. Journal of Policy and Practice in Intellectual Disabilities, 12, 79-89.

Morán, L., Gómez, L. E. y Alcedo, M. A. (2019). Inclusión social y autodeterminación: los retos en la calidad de vida de los jóvenes con autismo y discapacidad. Siglo Cero, 50(3), 2946. https://doi.org/ 10.14201/scero20195032946

Naciones Unidas. (2006). United Nations Convention on the Rights of Persons with Disability. https://www.un.org/development/desa/disabilities/convention-on-the-rights-ofpersons-with-disabilities.html

Navas, P., Amor, A. M., Crespo, M., Wolowiec, Z. y Verdugo, M. Á. (2020). Supports for people with intellectual and developmental disabilities during the CovID-19 pandemic from their own perspective. Research in Developmental Disabilities, 108, 103813.

Navas, P., Gómez, L. E., Verdugo, M. Á. y Schalock, R. L. (2012). Derechos de las personas con discapacidad intelectual: implicaciones de la Convención de Naciones Unidas. Siglo Cero, 43(3), 7-28.

Navas, P., Verdugo, M. Á., Amor, A. M., Crespo, M. y Martínez, S. (2020). Covid-19 y discapacidades intelectuales y del desarrollo: impacto del confinamiento desde la perspectiva de las personas, sus familiares y los profesionales y organizaciones que prestan apoyo. Plena inclusión España e Instituto Universitario de Integración en la Comunidad (INICO), Universidad de Salamanca.

Nussbaum, M. C. (2011). Creating capabilities: the human development approach. Belknap Press of Harvard University.

Onken, S. J. (2018). Mental health consumer concept mapping of supported community. Evaluation and Program Planning, 71, 36-45. https://doi.org/10.1016/j.evalprogplan.2018.08.001

Parmenter, T. R. (1992). Quality of life of people with developmental disabilities. International Review of Research in Mental Retardation, 18, 247-287.

Qian, X., Larson, S. A., Ticha, R., Stancliffe, R. y Pettingell, S. L. (2019). Active support training, staff assistance, and engagement of individuals with intellectual and developmental disabilities in the United States: randomized controlled trial. American Journal on Intellectual and Developmental Disabilities, 124, 157-173. https://doi.org/10.1352/1944-7558124.2.157

Raphael, D., Brown, I., Renwick, R. y Rootman, I. (1996). Assessing the quality of life of persons with developmental disabilities: description of a new model, measuring instruments, and initial findings. International Journal of Disability Development and Education, 43, 25-42. https://doi.org/10.1080/0156655960430103

Reinders, H. S. y Schalock, R. L. (2014). How organizations can enhance the quality of life of their clients and assess their results: the concept of quality of life enhancement. American Journal on Intellectual and Developmental Disabilities, 119, 291-302. https://doi. org/10.1352/1944-7558-119.4.291

Sánchez-Gómez, V., López, M., Amor, A. M. y Verdugo, M. Á. (2020). Apoyos para la calidad de vida de escolares con y sin discapacidad: revisión de literatura. Revista Internacional de Educación para la Justicia Social, 9(2), 327-349. https://doi.org/10.15366/riejs2020.9.2.016

Ediciones Universidad de Salamanca / CC BY-NC-ND

Siglo Cero, vol. 52 (3), 2021, julio-septiembre, pp. 9-28 
EL MODELO DE CALIDAD DE VIDA Y APOYOS:

LA UNIÓN TRAS VEINTICINCO AÑOS DE CAMINOS PARALELOS

M. Á. VERDUGO, R. L SCHALOCK Y L. E. GÓMEZ

Schalock, R. L., Borthwick-Duffy, S. A., Bradley, V. J., Buntinx, W. H. E., Coulter, D., Craig, E. M., Gomez, S. C., Lachapelle, Y., Luckasson, R., Reeve, A., Shogren, K. A., Snell, M. E., Spreat, S., Tassé, M. J., Thompson, J. R., Verdugo, M. Á., Wehmeyer, M. y Yeager, M. H. (2010). Intellectual disability. Definition, classification, and systems of supports (11th Edition). American Association on Intellectual and Developmental Disabilities. [Traducción al español por M. Á. VERDUGO, Definición, clasificación y sistemas de apoyos. Alianza, 2011].

Schalock, R. L., Brown, I., Brown, R., Cummins, R. A., Felce, D., Matikka, L., Keith, K. D. y PARmenter, T. (2002). Conceptualization, measurement, and application of quality of life for persons with intellectual disability: report of an international panel of experts. Mental Retardation, 40, 457-470. https://doi.org/10.1352/1944-7558-119.4.291

Schalock, R. L., Gómez, L. E., Verdugo, M. Á. y Reinders, H. S. (2016). Moving us toward a theory of individual quality of life. American Journal on Intellectual and Developmental Disabilities, 121, 1-12. https://doi.org/10.1352/1944-7558-121.1.1

SCHALOCK, R. L. y KeITH, K. D. (Eds.). (2016). Cross-cultural quality of life: enbancing the lives of people with intellectual disabilities. American Association on Intellectual and Developmental Disabilities.

SCHAlOCK, R. L. y LuCKASSON, R. (2020). Person-centered outcome evaluation: aligning a modern understanding of intellectual and developmental disabilities, individualized supports, valued outcomes, and meaningful impacts. Manuscrito enviado para publicación.

SCHAlOCK, R. L., LuCKasson, R. y TAssé, M. J. (2019). The contemporary view of intellectual and developmental disabilities. Implications for psychologists. Psicothema, 31, 223-228. https://doi.org/10.7334/psicothema2019.119

SCHAlOCK, R. L., LuCKasson, R. y TAssé, M. J. (en prensa). Ongoing transformation in the field of IDD: taking action for future progress. Intellectual and Developmental Disabilities.

SChalock, R. L., Luckasson, R. y TAssé, M. J. (2021). Intellectual disability: definition, diagnosis, classification, and planning supports (12 ${ }^{\mathrm{a}}$ Edition). AAIDD.

Schalock, R. L., Luckasson, R., Tassé, M. J. y Verdugo, M. Á. (2018). A holistic theoretical approach to intellectual disability: going beyond the four current perspectives. Intellectual and Developmental Disabilities, 56(2), 79-89. https://doi.org/10.1352/1934-9556-56.2.79

Schalock, R. L., Thompson, J. R. y Tassé, M. J. (2018). A systematic approach to personal support plans. American Association on Intellectual and Developmental Disabilities.

SCHAlock, R. L. y Verdugo, M. Á. (2002). Handbook on quality of life for human service practitioners. American Association on Mental Retardation. [Calidad de vida. Manual para profesionales de la educación, salud y servicios sociales. Alianza, 2003].

SCHAlOCK, R. L. y Verdugo, M. Á. (2012). A leadership guide to redefining intellectual and developmental disabilities organizations: eight successful change strategies. Brookes Publishing Company. [El cambio en las organizaciones de discapacidad. Estrategias para superar sus retos y hacerlo realidad. Guía de liderazgo. Alianza, 2013].

Schalock, R. L. y Verdugo, M. Á. (2013). The transformation of disabilities organizations. Intellectual and Developmental Disabilities, 51, 273-286. https://doi.org/ 10.1352/19349556-51.4.273

SCHALOCK, R. L. y VERDUGO, M. Á. (2019). International developments influencing the field of intellectual and developmental disabilities. En K. A. KeITH (Ed.), Cross-cultural psychology: contemporary themes and perspectives ( ${ }^{\text {nd }}$ Ed.) (pp. 309-323). Wiley-Blackwell.

Schalock, R. L., Verdugo, M. Á. y Gómez, L. E. (2011). Evidence-based practices in the field of intellectual and developmental disabilities: an international consensus approach.

Ediciones Universidad de Salamanca / CC BY-NC-ND

Siglo Cero, vol. 52 (3), 2021, julio-septiembre, pp. 9-28 
EL MODELO DE CALIDAD DE VIDA Y APOYOS:

LA UNIÓN TRAS VEINTICINCO AÑOS DE CAMINOS PARALELOS

M. Á. VERDUGO, R. L SCHALOCK Y L. E. GÓMEZ

Evaluation and Program Planning, 34, 273-282. https://doi.org/10.1016/j.evalprogplan.2010.10.004

Schalock, R. L., Verdugo, M. Á. y Gómez, L. E. (2020). The quality of life supports paradigm: its description and application in the field of intellectual and developmental disabilities. Journal of Policy and Practice in Intellectual Disabilities. Manuscrito enviado para publicación.

Schalock, R. L., Verdugo, M. Á. y van Loon, J. (2018). Understanding organization transformation in evaluation and program planning. Evaluation and Program Planning, 67, 5360. https://doi.org/ 10.1016/j.evalprogplan.2017.11.003

Shogren, K. A., Luckasson, R. y Schalock, R. L. (2020). Using a multidimensional model to analyze context and enhance personal outcomes. Intellectual and Developmental Disabilities, 58, 95-110. https://doi.org/10.1352/1934-9556-58.2.95

Shogren, K. A., Luckasson, R. y Schalock, R. L. (en prensa). Leveraging the power of context in disability policy development, implementation, and evaluation: multiple applications to enhance personal outcomes. Journal of Disability Policy Studies.

Shogren, K. A., Wehmeyer, M. L., Uyanik, H. y Heidrich, M. (2017). Development of the support decision making inventory system. Intellectual and Developmental Disabilities, 47, 220-233. https://doi.org/ 10.1352/1934-9556-55.6.432

Stancliffe, R. J., Arnold, S. R. C. y Riches, V. C. (2016). The supports paradigm. En R. L. Schalock y K. D. KeITH (Eds.), Cross-cultural quality of life: enhancing the lives of people with intellectual disabilities (pp. 133-142). American Association on Intellectual and Developmental Disabilities.

Summers, J. A., Poston, D., Turnbull, A., Marquis, J., Hoffman, L. Mannan, H. y Wang, M. (2005). Conceptualizing and measuring family quality of life. Journal of Intellectual Disability Research, 49, 777-783. https://doi.org/ 10.1111/j.1365-2788.2005.00751.x /

Thompson, J. R., Bradley, V. J., Buntinx, W., Schalock, R. L., Shogren, K. A., Snell, M. E., Wehmeyer, M. L., Borthwick-Duffy, S., Coulter, D. L., Craig, E. P. M., Gómez, S. C., Lachapelle, Y., Luckasson, R. A., Reeve, A., Spreat, S., Tassé, M. J., Verdugo, M. Á. y Yeager, M. H. (2009). Conceptualizing supports and the support needs of people with intellectual disability. Intellectual and Developmental Disabilities, 47, 135- 146. https:// doi.org/10.1352/1934-9556-47.2.135

Thompson, J. R., Bryant, B. R., Schalock, R. L., Shogren, K. A., Tassé, M. J., Wehmeyer, M. L., Campbell, E. M., Craig, E. M., Hughes, C. y Rotholz, D. A. (2015). Supports Intensity Scale-Adult Version User's Manual. American Association on Intellectual and Developmental Disabilities.

Thompson, J. R., Schalock, R. L., Agosta, J., Teninty, L. y Fortune, J. (2014). How the supports paradigm is transforming the developmental disabilities service system. Inclusion, 2, 86-99. https://doi.org/10.1352/2326-6988-2.2.86

Thompson, J. R., Wehmeyer, M. L., Hughes, C., Shogren, K. A., Seo, H., Little, T. D., Schalock, R. L., Realon, R. E., Copeland, S. R., Patton, J. R. y Tassé, M. J. (2016). Supports Intensity Scale-Children's Version (SIs-C). American Association on Intellectual and Developmental Disabilities.

Van Hecke, N., Claes, C., Vanderplasschen, W., De Maeyer, J., De Witte, N. y VandevelDE, S. (2018). Conceptualisation and measurement of quality of life based on Schalock and Verdugo's model: a cross-disciplinary review of the literature. Social Indicators Research, 137, 335-351. https://doi.org/10.1007/s11205-017-1596-2

Ediciones Universidad de Salamanca / CC BY-NC-ND

Siglo Cero, vol. 52 (3), 2021, julio-septiembre, pp. 9-28 
Verdugo, M. Á., Aguayo, V., Arias, V. B. y García-Domínguez, L. (2020). A Systematic review of the assessment of support needs in people with intellectual and developmental disabilities. International Journal of Environmental Research and Public Health, 17(24), 9494.

Verdugo, M. Á., Arias, B., Guillén, V. M., Amor, A., Aguayo, V., Vicente, E. y Jiménez, P. (2021). Escala SIs-C de Evaluación de las Necesidades de Apoyo para niños y adolescentes con discapacidades intelectuales y del desarrollo. Universidad de Salamanca, Publicaciones del INICO.

Verdugo, M. Á., Navas, P., Gómez, L. E. y Schalock, R. L. (2012). The concept of quality of life and its role in enhancing human rights in the field of intellectual disability. Journal of Intellectual Disability Research, 56, 1036-1045. https://doi.org/ 10.1111/j.1365-2788.2012. 01585.x

Vicente, E., Mumbardó, C., Coma, T., Verdugo, M. Á. y Giné, C. (2018). Autodeterminación en personas con discapacidad intelectual y del desarrollo: revisión del concepto, su importancia y retos emergentes. Revista Española de Discapacidad, 6(2), 7-25.

Vicente, E., Verdugo, M. Á., Guillén, V. M., Martínez-Molina, A., Gómez, L. E. e Ibáñez, A. (2020). Advances in the assessment of self-determination: internal structure of a scale for people with intellectual disabilities aged 11 to 40. Journal of Intellectual Disability Research, 64(9), 700-712.

Wehmeyer, M. L. (Ed.). (2013). The Oxford handbook of positive psychology and disability. Oxford University Press.

Zuna, N., Summers, J. A., Turnbull, A. P., Hu, X. y Xu, S. (2010). Theorizing about family quality of life. En R. KoBER (Ed.), Enhancing the quality of life of people with intellectual disability: from theory to practice (pp. 241-278). Springer. 